\title{
Necessary Conditions for Boundedness of Translation Operator in de Branges Spaces
}

\section{Carlo Bellavita ${ }^{1}$}

Received: 6 March 2021 / Accepted: 4 July 2021 / Published online: 21 July 2021

(c) The Author(s) 2021

\begin{abstract}
The translation operator is bounded in the Paley-Wiener spaces and, more generally, in the Bernstein spaces. The goal of this paper is to find some necessary conditions for the boundedness of the translation operator in the de Branges spaces, of which the Paley-Wiener spaces are special cases. Indeed, if the vertical translation operator $T_{\tau}$ defined on the de Branges space $\mathcal{H}(E)$ is bounded, then a suitably defined measure $d \mu(z)$ is a Carleson measure for the associated model space $K(\Theta)$. This relation allows us to state necessary conditions for the boundedness of the vertical translation $T_{\tau}$. Finally, similar results are also obtained for the horizontal translation $T_{\sigma}$.
\end{abstract}

Keywords de Branges spaces · Carleson measures for model spaces

Mathematics Subject Classification 30H45 · 30H99

\section{Introduction}

The purpose of this paper is to look for necessary conditions for the boundedness of the translation operator in the de Branges spaces $\mathcal{H}(E)$. We focus on the vertical translation operator $T_{\tau}$, which is defined as

$$
\begin{aligned}
& T_{\tau}: \quad \operatorname{Dom}\left\{T_{\tau}\right\} \subseteq \mathcal{H}(E) \rightarrow \mathcal{H}(E) \quad f(z) \rightarrow T_{\tau}[f](z):=f(z+i \tau) \\
& \quad z \in \mathbb{C}, \tau>0 .
\end{aligned}
$$

Communicated by Javad Mashreghi.

This article is part of the topical collection "Reproducing kernel spaces and applications" edited by Daniel Alpay.

$\triangle$ Carlo Bellavita

carlo.bellavita@unimi.it

1 Department of Mathematics, Università degli Studi di Milano, Milan, Italy 
Our interest for this problem has got a double origin. Firstly, we know that the vertical translation operator $T_{\tau}$ is bounded in the Paley-Wiener spaces, that is, $\mathcal{H}\left(e^{-i a z}\right)$, and more generally, in the Bernstein spaces [8]. This fact makes us wonder if the vertical translation operator $T_{\tau}$ is bounded also in other de Branges spaces as well.

Secondly, translation operator is closely related to the differentiation operator. The Bernstein inequality and the boundedness of the differentiation operator has been already widely studied by Dyakonov [6], and by Baranov [2-4] among the other papers.

We associate to the vertical translation operator the following measure defined in $\mathbb{C}^{+}$

$$
d \mu(z):=\sum_{n} \pi \frac{\delta_{t_{n}+i \tau}(z)}{\varphi^{\prime}\left(t_{n}\right)}\left|\frac{E\left(t_{n}+i y\right)}{E\left(t_{n}\right)}\right|^{2},
$$

where the values of $\left\{t_{n}\right\}$ will be specified later. Our first result is obtained in the second section, where we prove that $d \mu(z)$ is a Carleson measure for the associated model space $\mathcal{K}(\Theta)$ if $T_{\tau}$ is bounded. It means that the characterization of Carleson measures in model spaces is meaningful for the boundedness of $T_{\tau}$. About this topic, the first fundamental results were obtained by A.L. Vol'berg and S.R. Treil' in [15], where they gave a sufficient condition for a measure to be a Carleson measure in $\mathcal{K}(\Theta)$ and a necessary and sufficient condition to be a Carleson measure in $\mathcal{K}(\Theta)$ when $\Theta$ is a one component inner function. Subsequently A.B. Aleksandrov gave a different proof of the same theorems in [1].

More recently, a complete characterization of Carleson measures in model spaces has been obtained [7,9-11]. Thanks to these results, in the second section we find also the necessary and sufficient condition the measure $d \mu(z)$ needs to satisfy to be a Carleson measure in $\mathcal{K}(\Theta)$ according to [9].

In the third section we find another necessary condition for the boundedness of $T_{\tau}$. We prove that if $T_{\tau}$ is bounded in $\mathcal{H}(E)$, then the Hermite-Biehler function $E$ satisfies the following condition:

$$
\sup _{x \in \mathbb{R}}\left|\frac{E(x+i \tau)}{E(x)}-\frac{\overline{E(x-i \tau)}}{E(x)}\right| \frac{1}{\tau \varphi^{\prime}(x)} \leq \tilde{C} .
$$

In order to do this, we link the measure $d \mu(z)$ to an infinite matrix $\Gamma$, which defines a bounded operator in $\ell^{2}(\mathbb{Z})$.

Finally, in the fourth section we study the horizontal translation operator $T_{\sigma}$; it is defined in the same way:

$$
\begin{aligned}
& T_{\sigma}: \quad \operatorname{Dom}\left\{T_{\sigma}\right\} \subseteq \mathcal{H}(E) \rightarrow \mathcal{H}(E) \quad f(z) \rightarrow T_{\sigma}[f](z):=f(z+\sigma) \\
& \quad z \in \mathbb{C}, \sigma>0 .
\end{aligned}
$$

As already done for $T_{\tau}$, we associate a Carleson measure to $T_{\sigma}$, which, this time, is supported on the real line. This fact allows us to give an easier necessary condition that the horizontal translation operator must satisfy in order to be bounded on $\mathcal{H}(E),(4.5)$. 
The Condition (4.5) holds only when the derivative of the phase of the Hermite-Biehler function $E$ is lower and upper bounded on $\mathbb{R}$.

Let us first recall some definitions concerning the de Branges spaces $\mathcal{H}(E)$. For an extended description of $\mathcal{H}(E)$, one can study the book [5] and the papers [13,14].

An entire functions $E$ belongs to the Hermite-Biehler class if it satisfies the inequality

$$
|E(z)|>|E(\bar{z})| \quad z \in \mathbb{C}^{+}
$$

We define $\mathcal{H}(E)$ in the following way:

$$
\mathcal{H}(E):=\left\{f \text { entire }: \frac{f}{E} \in H^{2}\left(\mathbb{C}^{+}\right), \quad \frac{f^{\#}}{E} \in H^{2}\left(\mathbb{C}^{+}\right)\right\},
$$

where $H^{2}\left(\mathbb{C}^{+}\right)$is the Hardy space of the upper half plane.

Using the reproducing kernel of the Hardy space $H^{2}\left(\mathbb{C}^{+}\right)$, we also define the reproducing kernel $k_{w}$ of the de Branges space $\mathcal{H}(E)$ [5, Problem 50],

$$
k_{w}(z)= \begin{cases}\frac{E^{\#}(z) E(\bar{w})-E(z) E^{\#}(\bar{w})}{2 \pi i(z-\bar{w})}, & w \neq \bar{z} \\ \frac{E^{\# \prime}(z) E(z)-E^{\prime}(z) E^{\#}(z)}{2 \pi i}, & w=\bar{z}\end{cases}
$$

Moreover, we associate to the Hermite-Biehler function $E$ the phase function $\varphi$, that is the $C^{\infty}(\mathbb{R})$ function such that

$$
e^{i \varphi(x)} E(x):=|E(x)| \quad \forall x \in \mathbb{R} .
$$

The following relationship between $\varphi$ and $k_{w}$ holds:

$$
k_{x}(x):=|E(x)|^{2} \frac{\varphi^{\prime}(x)}{\pi} \quad \text { when } x \in \mathbb{R} .
$$

Finally, we note that the de Branges space $\mathcal{H}(E)$ is unitary equivalent to the model space $\mathcal{K}(\Theta)$ where

$$
\Theta:=\frac{E^{\#}}{E}
$$

and the function

$$
K_{w}(z): \frac{1}{\pi} \frac{1-\overline{\Theta(w)} \Theta(z)}{z-\bar{w}} \quad w \in \mathbb{C}^{+}
$$

is the reproducing kernel of $K(\Theta)$.

We say that $x \equiv_{\pi} \alpha$ if there is $k \in \mathbb{Z}$ such that $x=\alpha+k \pi$. A positive function $f(x) \asymp 1$ if there are two positive constants $C, B$ such that $0<C \leq f(x) \leq B<\infty$, for every $x \in \mathbb{R}$. 


\section{Vertical Translation, Carleson Measure and Two Weighted Hilbert Transform}

In this section we would like to show that the problem of the boundedness of vertical translation operator $T_{\tau}$ in the de Branges space $\mathcal{H}(E)$ can be reformulated as a problem concerning Carleson measures in the model space $\mathcal{K}(\Theta)$. Next, we will show that this problem is equivalent to the boundedness of the two weighted Hilbert transform. The measures involved will be purely atomic.

Notation Given the de Branges space $\mathcal{H}(E)$, we can choose families of points $\left\{t_{n}^{\alpha}\right\}_{n \in \mathbb{Z}} \subset \mathbb{R}$ with $0 \leq \alpha<\pi$ so that the set of reproducing kernel $k_{t_{n}^{\alpha}}(z)$ is a basis of $\mathcal{H}(E)$ for every $\alpha$, except at most one $\alpha$ [5].

Theorem 2.1 If the operator $T_{\tau}$ is bounded in $\mathcal{H}(E)$, then the measure $d \mu(z)$ in $(1.2)$ is a Carleson measure for $\mathcal{K}(\Theta)$, the associated model space.

Proof Let us consider

$$
\left\{t_{n}\right\}_{n \in \mathbb{Z}} \subset \mathbb{R} \text { such that } \varphi\left(t_{n}\right) \equiv_{\pi} 0 .
$$

We make the following computations:

$$
\begin{aligned}
C\|f\|_{\mathcal{H}}^{2} & \geq\left\|T_{i \tau} f\right\|_{\mathcal{H}}^{2} \geq \sum_{n \in \mathbb{Z}}\left|\left\langle T_{i \tau} f, \frac{k_{t_{n}}}{\left\|k_{t_{n}}\right\|_{\mathcal{H}}}\right\rangle_{\mathcal{H}}\right|^{2}=\sum_{n \in \mathbb{Z}}\left|\frac{f\left(t_{n}+i \tau\right)}{\left\|k_{t_{n}}\right\|_{\mathcal{H}}}\right|^{2} \\
& =\sum_{n \in \mathbb{Z}}\left|\left\langle f, \frac{k_{t_{n}+i \tau}}{\left\|k_{t_{n}}\right\|_{\mathcal{H}}}\right\rangle_{\mathcal{H}}\right|^{2}=\int_{\mathbb{C}^{+}}|f(z)|^{2}\left(\sum_{n} \frac{\delta_{t_{n}+i y}(z)}{\left\|k_{t_{n}}\right\|_{\mathcal{H}}^{2}}\right) .
\end{aligned}
$$

Moving to the model space $\mathcal{K}(\Theta) \ni F:=f / E$ when $f \in \mathcal{H}(E)$,

$$
C\|F\|_{\mathcal{K}}^{2} \geq \int_{\mathbb{C}^{+}}|F(z)|^{2}\left(\sum_{n} \pi \frac{\delta_{t_{n}+i \tau}(z)}{\varphi^{\prime}\left(t_{n}\right)}\left|\frac{E\left(t_{n}+i y\right)}{E\left(t_{n}\right)}\right|^{2}\right),
$$

that is

$$
\mathcal{K}(\Theta) \hookrightarrow L^{2}(d \mu) \text { continuously }
$$

Remark 2.2 We recall that, a priori, there could exists $0 \leq \alpha<\pi$ such that $\operatorname{dim}\left(\mathcal{H}(E) \ominus \overline{\operatorname{Span}}_{t_{n}}\left\{k_{t_{n}}\right\}\right)=1,[5]$.

Remark 2.3 In the proof of the above theorem, if we consider the points

$$
\left\{t_{n}^{\alpha}\right\}_{n \in \mathbb{Z}} \subset \mathbb{R} \text { such that } \varphi\left(t_{n}\right) \equiv_{\pi} \alpha \text {, for every } 0 \leq \alpha<\pi \text {, }
$$


the measure

$$
d \mu^{\alpha}(z):=\sum_{n} \pi \frac{\delta_{t_{n}^{\alpha}+i \tau}(z)}{\varphi^{\prime}\left(t_{n}^{\alpha}\right)}\left|\frac{E\left(t_{n}^{\alpha}+i y\right)}{E\left(t_{n}^{\alpha}\right)}\right|^{2}
$$

is still a Carleson measure for $\mathcal{K}(\Theta)$.

Notation To simplify the notation, we would say that $E\left(\Theta, \mu^{\alpha}\right)$ is the immersion operator from $K(\Theta)$ to $L^{2}\left(d \mu^{\alpha}\right)$. In Theorem 2.1, we proved that the operator $E\left(\Theta, \mu^{\alpha}\right)$ is continuous.

Before providing the necessary condition for the boundedness of $T_{\tau}$, in the rest of this section we want to prove the necessary and sufficient condition that the measure $d \mu^{\alpha}$ must satisfy in order to be a Carleson measure for $\mathcal{K}(\Theta)$ [9]. From now on we omit the letter $\alpha$ as our calculations work for any $0 \leq \alpha<\pi$.

First of all let us prove an equivalent description for $K(\Theta)$.

Theorem 2.4 Let

$$
d \sigma(z):=\sum_{n \in \mathbb{Z}} \pi \frac{\delta_{t_{n}}(z)}{\varphi^{\prime}\left(t_{n}\right)}
$$

The operator $H_{0}$, defined as

$$
H_{0}(f)(z):=(1-\Theta(z)) \int_{\mathbb{R}} \frac{f(t)}{t-z} \frac{1}{\pi} d \sigma(t)
$$

is a unitary operator from $L^{2}(d \sigma)$ to $\mathcal{K}(\Theta)$.

Proof This theorem is the Nyquist-Shannon sampling theorem for Model spaces [5, 12]. Indeed for every $f \in \mathcal{K}(\Theta)$

$$
f(z):=\sum_{n \in \mathbb{Z}}\left\langle f, \frac{K_{t_{n}}}{\left\|K_{t_{n}}\right\|}\right\rangle \frac{K_{t_{n}}}{\left\|K_{t_{n}}\right\|}=\sum_{n \in \mathbb{Z}} \frac{\pi f\left(t_{n}\right)}{\varphi^{\prime}\left(t_{n}\right)} K_{t_{n}}(z)=\sum_{n \in \mathbb{Z}} \frac{\pi f\left(t_{n}\right)}{\varphi^{\prime}\left(t_{n}\right)} \frac{1-\Theta(z)}{\pi\left(z-t_{n}\right)} .
$$

Indeed we choose $t_{n}$ so that $\Theta\left(t_{n}\right)=1$.

The boundedness of $E(\Theta, \mu)$ is equivalent to the boundedness of the two weighted Hilbert transform $H_{\tau}$. 
Theorem 2.5 The immersion operator $E(\Theta, \mu)$ is bounded if and only if the two weighted Hilbert transform $H_{\tau}$ from $L^{2}(d \sigma) \rightarrow L^{2}(d \tau)$ is continuous, where

$$
\begin{aligned}
d \sigma(z) & :=\sum_{n \in \mathbb{Z}} \pi \frac{\delta_{t_{n}}(z)}{\varphi^{\prime}\left(t_{n}\right)} \\
d \tau(z) & :=\sum_{n \in \mathbb{Z}} \pi\left|1-\Theta\left(t_{n}+i \tau\right)\right|^{2}\left|\frac{E\left(t_{n}+i \tau\right)}{E\left(t_{n}\right)}\right|^{2} \frac{\delta_{t_{n}+i \tau}(z)}{\varphi^{\prime}\left(t_{n}\right)} \\
H_{\tau}(f)(z) & :=\int_{\mathbb{R}} \frac{f(t)}{t-z} d \sigma(t) \quad f \in L^{2}(d \sigma) .
\end{aligned}
$$

Proof Let us suppose that $H_{\tau}: L^{2}(d \sigma) \rightarrow L^{2}(d \tau)$ is bounded. Then we have that

$$
\begin{aligned}
& \int_{\mathbb{R}}\left|(1-\Theta(z)) H_{\tau}(f)(z)\right|^{2} \sum_{n \in \mathbb{Z}} \pi\left|\frac{E\left(t_{n}+i \tau\right)}{E\left(t_{n}\right)}\right|^{2} \frac{\delta_{t_{n}+i \tau}(z)}{\varphi^{\prime}\left(t_{n}\right)} \\
& =\int_{\mathbb{R}}\left|H_{\tau}(f)(z)\right|^{2} d \tau(z) \leq \mathcal{C}^{2}\|f\|_{L^{2}(\sigma)}^{2} .
\end{aligned}
$$

For any $g \in \mathcal{K}(\Theta)$, let $g=H_{0} f$ according to (2.2); the above inequality says that

$$
\int_{\overline{\mathbb{C}^{+}}}|g(z)|^{2} \sum_{n \in \mathbb{Z}} \pi\left|\frac{E\left(t_{n}+i \tau\right)}{E\left(t_{n}\right)}\right|^{2} \frac{\delta_{t_{n}+i \tau}(z)}{\varphi^{\prime}\left(t_{n}\right)} \leq \mathcal{C}^{2}\|f\|_{L^{2}(\sigma)}^{2}=\mathcal{C}^{2}\|g\|_{K_{\Theta}}^{2} .
$$

Thus, we have that $\left\|H_{\tau}\right\|^{2} \leq \mathcal{C}^{2}$. Since the argument is completely reversible, we can say that $\left\|H_{\tau}\right\|=\mathcal{C}$ which proves the theorem.

Using the conclusive result of Lacey-Sawyer-Shen-Uriarte-Tuero-Wick [10] we can characterize when the two weights Hilbert transform $H_{\tau}$ is bounded.

We would like to apply the general theorem of [9] to our particular situation (2.3), (2.4) and (2.5). The first condition becomes

$$
\begin{aligned}
& \frac{\tau\left(Q_{I}\right)}{|I|} \times \int_{\mathbb{R} \backslash I} \frac{|I|}{(|I|+\operatorname{dist}(t, I))^{2}} \sigma(d t) \leq \mathscr{A}_{2} \\
& \left(\sum_{t_{n} \in Q_{I}} \pi \frac{\left|1-\Theta\left(t_{n}+\sigma\right)\right|^{2}}{\varphi^{\prime}\left(t_{n}\right)}\left|\frac{E\left(t_{n}+\sigma\right)}{E\left(t_{n}\right)}\right|^{2}\right) /|I| \\
& \quad \times\left(\sum_{t_{n} \notin I} \frac{|I|}{\left(|I|+\operatorname{dist}\left(t_{n}, I\right)\right)^{2}} \frac{\pi}{\varphi^{\prime}\left(t_{n}\right)}\right) \leq \mathscr{A}_{2} \\
& \left(\sum_{t_{n} \in Q_{I}} \pi \frac{\left(\sigma \varphi^{\prime}\left(\tilde{t}_{n}\right)\right)^{2}}{\varphi^{\prime}\left(t_{n}\right)}\left|\frac{E\left(t_{n}+\sigma\right)}{E\left(t_{n}\right)}\right|^{2}\right)
\end{aligned}
$$




$$
\times\left(\sum_{t_{n} \notin I} \frac{1}{\left(|I|+\operatorname{dist}\left(t_{n}, I\right)\right)^{2}} \frac{\pi}{\varphi^{\prime}\left(t_{n}\right)}\right) \leq \mathscr{A}_{2} .
$$

where we used Lagrange theorem

$$
\left|1-\Theta\left(t_{n}+\sigma\right)\right|=\left|\Theta\left(t_{n}\right)-\Theta\left(t_{n}+\sigma\right)\right|=\left|\sigma \Theta^{\prime}\left(\tilde{t_{n}}\right)\right|=|\sigma| \varphi^{\prime}\left(\tilde{t_{n}}\right) .
$$

The second condition becomes

$$
\begin{aligned}
& \frac{\sigma(I)}{|I|} \times \int_{\mathbb{C}^{+} \backslash Q_{I}} \frac{|I|}{\left(|I|+\operatorname{dist}\left(z, Q_{I}\right)\right)^{2}} \tau(d z) \leq \mathscr{A}_{2} \\
& \left(\sum_{t_{n} \in I} \frac{\pi}{\varphi^{\prime}\left(t_{n}\right)}\right) /|I| \\
& \quad \times\left(\sum_{t_{n} \notin I} \pi \frac{|I|}{\left(|I|+\operatorname{dist}\left(t_{n}+\sigma, I\right)\right)^{2}} \frac{\left|1-\Theta\left(t_{n}+\sigma\right)\right|^{2}}{\varphi^{\prime}\left(t_{n}\right)}\left|\frac{E\left(t_{n}+\sigma\right)}{E\left(t_{n}\right)}\right|^{2}\right) \leq \mathscr{A}_{2} \\
& \left(\sum_{t_{n} \in I} \frac{\pi}{\varphi^{\prime}\left(t_{n}\right)}\right) \\
& \quad \times\left(\sum_{t_{n} \notin I} \pi \frac{1}{\left(|I|+\operatorname{dist}\left(t_{n}+\sigma, I\right)\right)^{2}} \frac{\left|\sigma \varphi\left(\tilde{t_{n}}\right)\right|^{2}}{\varphi^{\prime}\left(t_{n}\right)}\left|\frac{E\left(t_{n}+\sigma\right)}{E\left(t_{n}\right)}\right|^{2}\right) \leq \mathscr{A}_{2} .
\end{aligned}
$$

where we used again Lagrange theorem. The third condition becomes

$$
\begin{aligned}
& \int_{Q_{I}}\left|H_{\sigma} 1_{I}(x)\right|^{2} \tau(d x) \leq \mathscr{T}^{2} \sigma(I) \\
& \quad \sum_{t_{l}+\sigma \in I}\left|\sum_{t_{n} \in I} \frac{1}{t_{l}+\sigma-t_{n}} \frac{\pi}{\varphi^{\prime}\left(t_{n}\right)}\right|^{2} \pi \frac{\left|1-\Theta\left(t_{l}+\sigma\right)\right|^{2}}{\varphi^{\prime}\left(t_{l}\right)}\left|\frac{E\left(t_{l}+\sigma\right)}{E\left(t_{l}\right)}\right|^{2} \\
& \leq \mathscr{T}^{2} \sum_{t_{n} \in I} \frac{\pi}{\varphi^{\prime}\left(t_{n}\right)} .
\end{aligned}
$$

Finally the fourth condition becomes 


$$
\begin{aligned}
& \int_{I}\left|H_{\tau} 1_{Q_{I}}(x)\right|^{2} \sigma(d x) \leq \mathscr{T}^{2} \tau\left(Q_{I}\right) \\
& \left.\left.\quad \sum_{t_{n} \in I}\left|\sum_{t_{l}+\sigma \in I} \frac{1}{t_{n}-t_{l}-\sigma} \pi \frac{\left|1-\Theta\left(t_{l}+\sigma\right)\right|^{2}}{\varphi^{\prime}\left(t_{l}\right)}\right| \frac{E\left(t_{l}+\sigma\right)}{E\left(t_{l}\right)}\right|^{2}\right|^{2} \frac{\pi}{\varphi^{\prime}\left(t_{n}\right)} \\
& \leq \mathscr{T}^{2} \sum_{t_{l}+\sigma \in I} \pi \frac{\left|1-\Theta\left(t_{l}+\sigma\right)\right|^{2}}{\varphi^{\prime}\left(t_{l}\right)}\left|\frac{E\left(t_{l}+\sigma\right)}{E\left(t_{l}\right)}\right|^{2} .
\end{aligned}
$$

Therefore we can state the following theorem.

Theorem 2.6 The measure $d \mu(z)$ defined in (1.2) is a Carleson measure for $\mathcal{K}(\Theta)$ if and only if the measure $d \sigma(z)$ in (2.3), $d \tau(z)$ in (2.4) and the two weighted Hilbert transform $H_{\tau}$ in (2.5) satisfy the conditions (2.6), (2.7), (2.8) and (2.9).

\section{Boundedness of $T_{\tau}$ and the Matrix $\Gamma$}

In this section we prove a necessary condition the Hemite-Biehler $E$ must satisfy if the vertical translation operator $T_{\tau}$ is bounded in $\mathcal{H}(E)$.

Indeed if $\left\|T_{\tau}\right\|<C$, then, due to Theorem 2.1,

$$
\begin{aligned}
C^{2}\left\|K_{t_{n}^{\alpha}}\right\|^{2} & \geq \sum_{l \in \mathbb{Z}}\left|\left\langle K_{t_{n}^{\alpha}}, K_{t_{l}^{\alpha}+i \tau}\right\rangle\right|^{2} \frac{\left|E\left(t_{l}^{\alpha}+i \tau\right)\right|^{2}}{\left\|k_{t_{l}^{\alpha}}\right\|^{2}} \\
& =\sum_{l \in \mathbb{Z}}\left|K_{t_{n}^{\alpha}}\left(t_{l}^{\alpha}+i \tau\right)\right|^{2} \frac{\left|E\left(t_{l}^{\alpha}+i \tau\right)\right|^{2}}{\left\|k_{t_{l}^{\alpha}}\right\|^{2}} \\
& =\sum_{l \in \mathbb{Z}}\left|k_{t_{n}^{\alpha}}\left(t_{l}^{\alpha}+i \tau\right)\right|^{2} \frac{1}{\left\|k_{t_{l}^{\alpha}}\right\|^{2}\left|E\left(t_{n}^{\alpha}\right)\right|^{2}} .
\end{aligned}
$$

This implies that $\forall n \in \mathbb{Z}$ and for every $0 \leq \alpha<\pi$

$$
\sum_{l \in \mathbb{Z}}\left|k_{t_{n}^{\alpha}}\left(t_{l}^{\alpha}+i \tau\right)\right|^{2} \frac{1}{\left\|k_{t_{n}^{\alpha}}\right\|^{2}\left\|k_{t_{l}^{\alpha}}\right\|^{2}} \leq C^{2}
$$

The above relation assure that there exists a family of uniformly bounded operators $\Gamma(\alpha)$ in $\ell^{2}(\mathbb{Z})$. 
Proposition 3.1 For every $0 \leq \alpha<\pi$, there exists an infinite matrix $\Gamma(\alpha)$

$$
\begin{gathered}
\Gamma(\alpha): \ell^{2}(\mathbb{Z}) \rightarrow \ell^{2}(\mathbb{Z}) \\
\Gamma(\alpha)\left[(c)_{n}\right]:=\left(\sum_{n \in \mathbb{Z}}\left\langle k_{t_{n}^{\alpha}}, k_{t_{l}^{\alpha}+i \tau}\right\rangle \frac{1}{\left\|k_{t_{n}^{\alpha}}\right\|\left\|k_{t_{l}^{\alpha}}\right\|} c_{n}\right)_{l}
\end{gathered}
$$

which acts in $\ell^{2}(\mathbb{Z})$, such that for every $0 \leq \alpha<\pi$

$$
\|\Gamma(\alpha)\| \leq C
$$

Proof First of all, thanks to Shannon sampling theorem for de Branges spaces [5], we know that for every $0 \leq \alpha<\pi$,

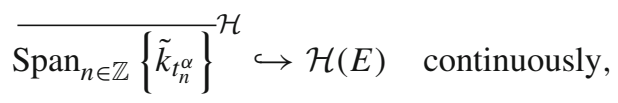

where $\tilde{k}_{w}$ is the normalized reproducing kernel of $\mathcal{H}(E)$. We can now identify in the canonical way the space $\overline{\operatorname{Span}_{n \in \mathbb{Z}}\left\{\tilde{k}_{t_{n}^{\alpha}}\right\}}$ with $\ell^{2}(\mathbb{Z})$ as $\left\langle\tilde{k}_{t_{n}}, \tilde{k}_{t_{m}}\right\rangle_{\mathcal{H}}=\delta_{n, m}$. Therefore we define the operator

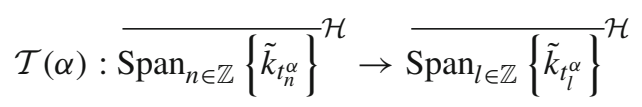

$$
\begin{aligned}
& \mathcal{T}(\alpha)\left(\tilde{k}_{t_{n}^{\alpha}}\right)(z):=\sum_{l \in \mathbb{Z}}\left\langle k_{t_{n}^{\alpha}}, k_{t_{l}^{\alpha}+i \tau}\right\rangle \frac{1}{\left\|k_{t_{n}^{\alpha}}\right\|\left\|k_{t_{l}^{\alpha}}\right\|} \tilde{k}_{t_{l}^{\alpha}}(z) .
\end{aligned}
$$

Thanks to estimate (3.1), for every $0 \leq \alpha<\pi$ the operator $\mathcal{T}(\alpha)$ is bounded. Consequently we can define the family of infinite matrices $\Gamma(\alpha)$ as in (3.2) such that $\|\Gamma(\alpha)\| \leq C$.

Remark 3.2 We can explicitly described the matrix $\Gamma(\alpha)$. Indeed

$\Gamma(\alpha)$

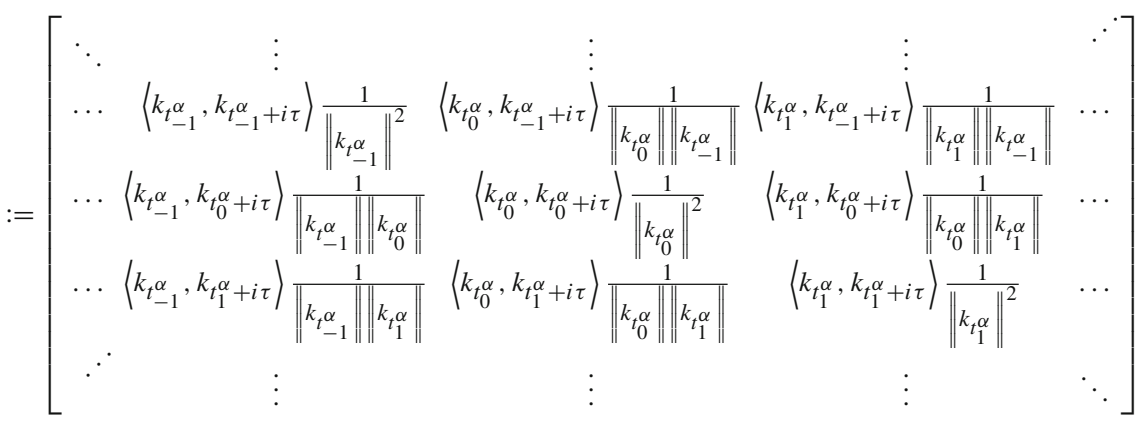


Of course, the diagonal elements are bounded; Indeed, for any $0 \leq \alpha<\pi$

$$
\left|\left\langle k_{t_{n}^{\alpha}}, k_{t_{n}^{\alpha}+i \tau}\right\rangle \frac{1}{\left\|k_{t_{n}^{\alpha}}\right\|^{2}}\right| \leq C \quad \forall n \in \mathbb{Z} .
$$

We are now ready to state the necessary condition the Hermite-Biehler function $E$ must satisfy when the vertical translation operator $T_{\tau}$ is bounded in $\mathcal{H}(E)$.

Theorem 3.3 Let us assume that the vertical translation operator $T_{\tau}$ is bounded in $\mathcal{H}(E)$. Then the Hermite-Biehler function E satisfies

$$
\sup _{x \in \mathbb{R}}\left|\frac{E(x+i \tau)}{E(x)}-\frac{\overline{E(x-i \tau)}}{E(x)}\right| \frac{1}{\tau \varphi^{\prime}(x)} \leq \tilde{C} .
$$

Proof As already explained in the above proposition, relation (3.5) holds. We explicitly compute (3.5) in order to obtain (3.6). Indeed

$$
\begin{aligned}
\tilde{C} & \geq\left|E\left(t_{n}^{\alpha}+i \tau\right) \overline{E\left(t_{n}^{\alpha}\right)}-\overline{E\left(t_{n}^{\alpha}-i \tau\right)} E\left(t_{n}^{\alpha}\right)\right| \frac{1}{\tau\left|E\left(t_{n}^{\alpha}\right)\right|^{2} \varphi^{\prime}\left(t_{n}^{\alpha}\right)} \\
& =\left|\frac{E\left(t_{n}^{\alpha}+i \tau\right)}{E\left(t_{n}^{\alpha}\right)}-\frac{\overline{E\left(t_{n}^{\alpha}-i \tau\right)}}{E\left(t_{n}^{\alpha}\right)}\right| \frac{1}{\tau \varphi^{\prime}\left(t_{n}^{\alpha}\right)} .
\end{aligned}
$$

By varying $0 \leq \alpha<\pi$ and $n \in \mathbb{Z}$, we get the estimate (3.6).

Remark 3.4 We point also that the estimate (3.6) can be rewritten as

$$
\sup _{x \in \mathbb{R}}\left|\frac{T_{\tau} E(x)}{E(x)}-\left(\frac{T_{\tau} E(x)}{E(x)}\right)^{\#}\right| \frac{1}{\tau \varphi^{\prime}(x)} \leq \tilde{C} .
$$

\section{Horizontal Translation and Carleson Measure}

In this section we prove a necessary condition for the boundedness of the horizontal translation $T_{\sigma}$. First of all we have the analogue of Theorem 2.1 with almost the same proof.

Theorem 4.1 If the operator $T_{\sigma}$ is bounded in $\mathcal{H}(E)$, the measure

$$
d \nu^{\alpha}(z):=\pi \sum_{n \in \mathbb{Z}} \frac{\delta_{t_{n}^{\alpha}+\sigma}(z)}{\varphi^{\prime}\left(t_{n}^{\alpha}\right)}\left|\frac{E\left(t_{n}^{\alpha}+\sigma\right)}{E\left(t_{n}^{\alpha}\right)}\right|^{2}
$$

is a Carleson measure for $\mathcal{K}(\Theta)$. 
Therefore if $T_{\sigma}$ is bounded the measure $d \nu^{\alpha}(z)$ is a Carleson measure for $\mathcal{K}(\Theta)$ and it is supported in $\mathbb{R}$.

The following theorem, proved in [2], will give us a necessary and sufficient condition the measure $d v^{\alpha}(z)$ of (4.1) must satisfy in order to be a Carleson measure for $\mathcal{K}(\Theta)$.

Theorem 4.2 Let E satisfy

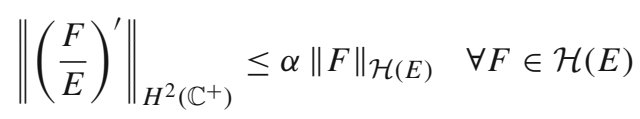

and let $\mu$ be a Borel measure on the line $\mathbb{R}$. Then (1) implies (2):

(1) There are constants $L, C_{L}>0$ such that $\mu(I)<C_{L} L$ for any interval I such that $|I| \leq L$

(2) The measure $\mu$ satisfies

$$
\int_{\mathbb{R}}\left|\frac{F}{E}\right|^{2} d \mu \leq C\|F\|_{\mathcal{H}}^{2} \quad \forall F \in \mathcal{H}(E) .
$$

Under the additional condition $\left|\varphi^{\prime}(t)\right|>0$, the condition (2) implies (1).

We can apply the above result to the measure $d v^{\alpha}(z)$ of (4.1).

Proposition 4.3 Let $\left|\varphi^{\prime}(x)\right| \asymp 1$. The measure

$$
d \nu^{\alpha}(z):=\pi \sum_{n \in \mathbb{Z}} \frac{\delta_{t_{n}^{\alpha}+\sigma}(z)}{\varphi^{\prime}\left(t_{n}^{\alpha}\right)}\left|\frac{E\left(t_{n}^{\alpha}+\sigma\right)}{E\left(t_{n}^{\alpha}\right)}\right|^{2}
$$

is a Carleson measure for $\mathcal{K}(\Theta)$ if and only if

$$
\sum_{t_{n}^{\alpha}+\sigma \in I} \frac{\pi}{\varphi^{\prime}\left(t_{n}^{\alpha}\right)}\left|\frac{E\left(t_{n}^{\alpha}+\sigma\right)}{E\left(t_{n}^{\alpha}\right)}\right|^{2} \leq C_{L} L
$$

for every interval $|I|<L$.

We can finally state the necessary condition the Hermite-Biehler function $E$ has to satisfy so that $T_{\sigma}$ is bounded.

Theorem 4.4 Let $\left|\varphi^{\prime}(x)\right| \asymp 1$. If the horizontal translation operator $T_{\sigma}$ is bounded, then

$$
\sup _{x \in \mathbb{R}}\left|\frac{E(x+\sigma)}{E(x)}\right| \leq C .
$$


Proof The Condition (4.5) comes directly from (4.4) by varying $\alpha \in[0, \pi$ ) and $n \in \mathbb{Z}$. Indeed, for every $x \in \mathbb{R}, x=\alpha+n \pi$ for some $0 \leq \alpha<\pi$ and $n \in \mathbb{Z}$. Consequently, since $C_{L}$ in (4.4) does not depend on $\alpha$,

$$
\begin{aligned}
\left|\frac{E(x+\sigma)}{E(x)}\right|^{2} & =\left|\frac{E\left(t_{n}^{\alpha}+\sigma\right)}{E\left(t_{n}^{\alpha}\right)}\right|^{2} \leq \frac{\sup _{x \in R}\left|\varphi^{\prime}(x)\right|}{\pi}\left(\frac{\pi}{\varphi^{\prime}\left(t_{n}^{\alpha}\right)}\left|\frac{E\left(t_{n}^{\alpha}+\sigma\right)}{E\left(t_{n}^{\alpha}\right)}\right|^{2}\right) \\
& \leq \frac{\sup _{x \in R}\left|\varphi^{\prime}(x)\right|}{\pi} C_{L} L .
\end{aligned}
$$

Funding Open access funding provided by Universitá degli Studi di Milano within the CRUI-CARE Agreement.

Data Availability All data generated or analysed during this study are included in this published article (and its supplementary information files).

\section{Declarations}

Conflict of interest The author declares that they no conflict of interest.

Open Access This article is licensed under a Creative Commons Attribution 4.0 International License, which permits use, sharing, adaptation, distribution and reproduction in any medium or format, as long as you give appropriate credit to the original author(s) and the source, provide a link to the Creative Commons licence, and indicate if changes were made. The images or other third party material in this article are included in the article's Creative Commons licence, unless indicated otherwise in a credit line to the material. If material is not included in the article's Creative Commons licence and your intended use is not permitted by statutory regulation or exceeds the permitted use, you will need to obtain permission directly from the copyright holder. To view a copy of this licence, visit http://creativecommons.org/licenses/by/4.0/.

\section{References}

1. Aleksandrov, A.B.: A simple proof of a theorem of Vol'berg and Treil' on the embedding of coinvariant subspaces of the shift operator. J. Math. Sci. 85, 1773-1778 (1997)

2. Baranov, A.D.: Differentiation in the Branges spaces and embedding theorems. J. Math. Sci. 101, 2881-2913 (2000)

3. Baranov, A.D.: Bernstein's inequality in the de Branges spaces and embedding theorems. Am. Math. Soc. Transl. 209, 21-49 (2003)

4. Baranov, A.D.: Estimate of the $L^{p}$-norms of derivatives in spaces of entire functions. J. Math. Sci. 129, 3927-3943 (2005)

5. de Branges, L.: Hilbert Spaces of Entire Functions. Prentice Hall, Englewood Cliffs (1968)

6. Dyakonov, K.M.: Entire functions of exponential type and model subspaces in $H^{p}$. J. Math. Sci. 71, 2222-2233 (1994)

7. Hytonen, T.: The two-weight inequality for the Hilbert transform with general measures. Proc. Lond. Math. Soc. 117(3), 483-526 (2018)

8. Levin, B.I.A., Lyubarskii, Y., Sodin, M., Tkachenko, V.: Lectures on Entire Functions. American Mathematical Society (1996)

9. Lacey, M.T., Sawyer, E.T., Shen, C.Y., Uriarte-Tuero, I.: Two-weight inequality for the Hilbert transform: a real variable characterization, I. Duke Math. J. 163(15), 2795-2820 (2014) 
10. Lacey, M., Sawyer, E.T., Shen, C.Y., Uriarte-Tuero, I., Wick, B.: Two Weight Inequalities for the Cauchy Transform from $\mathbb{R}$ to $\mathbb{C}^{+}$, arXiv : Complex Variables (2013)

11. Nazarov, F., Treil, S., Volberg, A.: Two weight estimate for the Hilbert transform and corona decomposition for non-doubling measures, arXiv : Analysis of PDEs (2010)

12. Poltoratski, A.: Toeplitz approach to problems of the uncertainty principle, CBMS Regional Conference Series in Mathematics, American Mathematical Society. Providence. Rhode Island 121 (2015)

13. Remling, C.: Schrödinger operators and de Branges spaces. J. Funct. Anal. 196(2), 395-426 (2002)

14. Romanov, R.: Canonical systems and de Branges spaces, arXiv : Complex Variables (2014)

15. Vol'berg, A.L., Treil', S.R.: Imbedding theorems for invariant subspaces of backward shift operator. J. Math. Sci. 42, 1562-1572 (1988)

Publisher's Note Springer Nature remains neutral with regard to jurisdictional claims in published maps and institutional affiliations. 